\title{
Strömgren photometry of 40 Harmonia, 45 Eugenia and 52 Europa
}

\author{
M.J. López-González and E. Rodríguez \\ Instituto de Astrofísica de Andalucía, CSIC, P.O. Box 3004, E-18080 Granada, Spain
}

Received June 16; accepted August 26, 1999

\begin{abstract}
The Asteroids 40 Harmonia, 45 Eugenia and 52 Europa have been studied photometrically. From their lightcurves synodic periods of $8^{\mathrm{h}} 54^{\mathrm{m}} 36^{\mathrm{s}}, 5^{\mathrm{h}} 42^{\mathrm{m}} 00^{\mathrm{s}}$ and $5^{\mathrm{h}} 37^{\mathrm{m}} 46^{\mathrm{s}}$, and maximum amplitudes of $0.15,0.12$ and 0.20 , have been deduced for 40 Harmonia, 45 Eugenia and 52 Europa, respectively. Improved solutions for the sense of rotation, sidereal period, pole orientation and shape properties are proposed.
\end{abstract}

Key words: minor planets, asteroids - techniques: photometric

\section{Introduction}

A very important part of the evolution of the solar system relates to the evolution of the minor planets. Amongst other characteristics, the fundamental attributes of an asteroid are its shape, the parameters that define its rotational motion, its taxonomic class and its possible colour and/or albedo variegation on its surface. Physical properties of asteroids such as their shapes, spin periods and spin axes can help in constructing our knowledge of their collisional evolution (Tedesco \& Zappala 1980; Farinella et al. 1981; Dermott et al. 1984).

Lightcurve observations of an asteroid are planned for more than one night to improve the accuracy of the period determination, to define the phase relation and absolute brightness as function of the solar phase angle and to determine possible albedo and colour variegation on the surface. Multiple observations of lightcurves from different aspects as an asteroid orbits provide information about spin period, axis orientation and body shape.

In this paper lightcurves of 40 Harmonia, 45 Eugenia and 52 Europa taken in 1997, for two observing periods are presented, one during September and the other during October, from the observatory of Sierra Nevada, Spain

Send offprint requests to: M.J. López-González e-mail: mariajose@iaa.es
(0.9 m Telescope). Our object is to increase the ecliptic longitude coverage of these asteroids to obtain a larger data base relating to their asteroidal lightcurves in order to improve future work on their rotational and shape properties.

Here, uvby Strömgren photometry for 40 Harmonia, 45 Eugenia and 52 Europa are obtained and used to derive phase coefficients correction, multiple scattering factors, Strömgren and Johnson zero-phase angle magnitudes and colour indices and to determine possible colour variegation of the surface of these asteroids during their rotational cycles. Results for rotational and shape parameters for these asteroids have also been obtained and compared with previous results.

\section{Observations}

The observations were carried out on different nights of September and October 1997 using the $90 \mathrm{~cm}$ telescope at Sierra Nevada Observatory, Spain. This telescope is equipped with a six channel uvby $\beta$ photometer for simultaneous measurements in uvby or in the $\mathrm{H}_{\beta}$ channels, respectively (Nielsen 1983), but only uvby measurements were collected during this observing run.

In order to make differential photometry, different sets of comparison stars were used during September and October. The comparison stars were chosen taking into account their spectral type (close to solar) and in the neighbourhood of the asteroids for better reduction of the data avoiding extinction problems. Each set of comparison stars contains one main comparison and one check star. During September observations $\mathrm{C} 1=\mathrm{SAO} 146842\left(V=7^{\mathrm{m}} 2, \mathrm{~F} 8\right)$ was used as the main comparison star and $\mathrm{C} 2=\mathrm{SAO}$ $146908\left(V=7{ }^{\mathrm{m}} 6, \mathrm{~F} 8\right)$ as a check star. During October observations $\mathrm{C} 3=\mathrm{SAO} 165708\left(V=6{ }^{\mathrm{m}} 4, \mathrm{G} 0\right)$ was used as the main comparison star and $\mathrm{C} 4=\mathrm{SAO} 165638\left(V=7^{\mathrm{m}} \cdot 7\right.$, G5) as a check star. In order to make the two sets of comparison stars compatible the main and the check stars of both sets were observed simultaneously during different nights. 
Table 1. Aspect data

\begin{tabular}{lccccc}
\hline $\begin{array}{l}\text { Date } \\
\text { (0 UT) }\end{array}$ & $\begin{array}{c}\text { Long } \\
(1950)\end{array}$ & $\begin{array}{c}\text { Lat } \\
(1950)\end{array}$ & $\begin{array}{c}\text { phase } \\
(\mathrm{deg})\end{array}$ & $\begin{array}{c}r \\
(\mathrm{AU})\end{array}$ & $\begin{array}{c}\Delta \\
(\mathrm{AU})\end{array}$ \\
\hline 40 Harmonia & & & & & \\
08 09 1997 & 352.11 & -7.65 & 4.92 & 1.16620 & 2.16443 \\
09 09 1997 & 351.86 & -7.67 & 4.56 & 1.16501 & 2.16431 \\
10 09 1997 & 351.61 & -7.68 & 4.23 & 1.16406 & 2.16419 \\
02 10 1997 & 346.36 & -7.59 & 10.43 & 1.20539 & 2.16231 \\
03 10 1997 & 346.17 & -7.56 & 10.91 & 1.21001 & 2.16225 \\
07 10 1997 & 345.47 & -7.45 & 12.76 & 1.23066 & 2.16208 \\
45 Eugenia & & & & & \\
08 09 1997 & 348.58 & -3.53 & 1.89 & 1.78740 & 2.79222 \\
09 09 1997 & 348.36 & -3.57 & 1.60 & 1.78771 & 2.79301 \\
10 09 1997 & 348.13 & -3.60 & 1.40 & 1.78831 & 2.79380 \\
02 10 1997 & 343.59 & -4.19 & 8.63 & 1.87130 & 2.81091 \\
03 10 1997 & 343.43 & -4.21 & 8.99 & 1.87811 & 2.81167 \\
07 10 1997 & 342.81 & -4.27 & 10.40 & 1.90779 & 2.81470 \\
52 Europa & & & & & \\
08 09 1997 & 352.22 & -7.19 & 3.22 & 2.23452 & 3.23064 \\
09 09 1997 & 352.03 & -7.22 & 2.98 & 2.23227 & 3.22979 \\
10 09 1997 & 351.83 & -7.25 & 2.76 & 2.23030 & 3.22893 \\
02 10 1997 & 347.54 & -7.60 & 6.69 & 2.25939 & 3.20973 \\
03 10 1997 & 347.37 & -7.61 & 7.00 & 2.26392 & 3.20885 \\
07 10 1997 & 346.71 & -7.61 & 8.25 & 2.28467 & 3.20528 \\
\hline
\end{tabular}

The observing and reduction procedure was standard, based on consecutive measurements of the selected main comparison star, check comparison star, asteroids and sky reading. During the observations reported here, neither of the comparison stars showed any sign of variability within 0.005 .

To transform our data into the standard uvby system we have used the same procedure described in Rodríguez et al. (1997). After differential magnitudes in the standard system were obtained for the asteroids with respect to one main comparison star $(\mathrm{C} 1=\mathrm{SAO} 146842)$ we correct them to unit distance from the sun and the earth and perform light-time corrections to all the observations. Then, we transform these differential magnitudes to absolute magnitudes using the absolute values of $\mathrm{C} 1, V=7 \mathrm{~m} 156$, $b-y=0.310, m_{1}=0.163$ and $c_{1}=0.413$, listed in the Hauck \& Mermilliod (1998) catalogue.

In addition, we obtained the following values of $V=$ $7^{\mathrm{m}} 656,6^{\mathrm{m}} 377,7^{\mathrm{m}} \cdot 750, b-y=0.290,0.548,0.589$, $m_{1}=0^{\mathrm{m}} 165,0.269,0^{\mathrm{m}} 345$, and $c_{1}=0.517,0.427$, 0. 357 for C2, C3 and C4, respectively, in good agreement with the values listed in Hauck \& Mermilliod (1998).

\section{Analysis}

In Table 1 the aspect data for each asteroid, the longitude and latitude relative to the ecliptic, the solar phase angle, the geocentric, $r$, and heliocentric, $\Delta$, distances, are listed for every day of observation.

\subsection{Photometry}

These observations were carried out during different nights of September and October when each asteroid had different phase angles, thus different magnitudes were observed. The mean reduced magnitudes observed for each uvby Strömgren filter, $\overline{u v b y}(1, \alpha)$, the average phase angle of the observations, $\bar{\alpha}$, and the range of phase angle covered, during September and October observations, are presented in Table 2. The observed Strömgren colour indices, $b-y$ and $u-b$, are also listed.

We have applied a linear phase correction to transform the observed uvby reduced magnitudes to zero-phase angle magnitudes, uvby $(1,0)$. No significative differences are found in the individual linear phase coefficients, $\beta_{\text {filter }}$, obtained using the different Strömgren filters. The slight differences found are, in all the cases, within the error bars of the determinations, but these slight differences could influence the colour indices when they are transformed to zero-phase angle. In order to preserve the colour indices measured, a mean linear phase coefficient, $\beta_{\mathrm{m}}$, that is more suitable for the observed magnitudes at phase angles greater than $7^{\circ}$ in all the Strömgren filters, is used to transform to zero-phase angle magnitudes. The magnitudes uvby $(1,0)$ are calculated as $u v b y(1, \alpha)=$ $u v b y(1,0)+\beta_{\mathrm{m}} \alpha$. This relation has been applied to the measurements taken during October when the solar phase angles were greater than $7^{\circ}$ for all the asteroids.

Lumme \& Bowell (1981a, 1981b) showed that the light observed at phase angle $\alpha$ relative to the luminosity at zero phase is decomposed into a single scattering part and a multiple scattering part, and the magnitudes at phase angle $\alpha$ can be expressed as $V(\alpha)=V\left(0^{\circ}\right)-2.5 \log ((1-Q) \phi(\alpha)+Q)$. Here, $\phi(\alpha)$ is the corresponding phase function for single scattered light and $Q$ is the multiple scattering factor. Lumme \& Bowell (1981b) found that $\phi(\alpha)$ can be expressed as $\phi(\alpha)=1-\sin \alpha /\left(0.124+1.407 \sin \alpha-0.758 \sin ^{2} \alpha\right)$ in the phase range $0^{\circ} \leq \alpha \leq 25^{\circ}$. We have used all the data measured from September to October, for each asteroid, to find the values of $Q_{\text {filter }}$ and $V_{\text {filter }}(0)$ which produce the best fit, for each filter, to the Lumme \& Bowell (1981b) relation.

The Strömgren magnitudes and colour indices obtained by linear analysis, $\overline{u v b y}(1,0)$, and those obtained by following the radiative transfer theory of Lumme \& Bowell (1981b), $\overline{u v b y}(0)$, are shown in Table 2. These Strömgren values are transformed into the $U B V$.Johnson system by using the transformation equations of Warren \& Hesser (1977) to transform the Strömgren $b-y$ and $u-b$ colour indices to Johnson $B-V$ and $U-B$ colour indices. As the " $y$ " standard magnitude obtained using the " $y$ " Strömgren filter is equivalent to the " $V$ " magnitude of the " $V$ " Johnson filter, knowing the $B-V$ and $U-B$ colour indices, the $B$ and $U$ magnitudes are calculated directly. The Johnson magnitudes and colour indices obtained are 
Table 2. Photometry

\begin{tabular}{|c|c|c|c|c|c|c|c|c|}
\hline $\begin{array}{c}\overline{u v b y}(1, \alpha) \\
(\mathrm{mag})\end{array}$ & $\begin{array}{c}\overline{u v b y}(1, \alpha) \\
(\mathrm{mag})\end{array}$ & $\begin{array}{c}\overline{u v b y}(1,0) \\
(\mathrm{mag})\end{array}$ & $\begin{array}{c}\overline{U B V}(1,0) \\
(\mathrm{mag})\end{array}$ & $Q$ factors & $\begin{array}{l}\overline{u v b y}(0) \\
(\mathrm{mag})\end{array}$ & $\begin{array}{c}\overline{U B V}(0) \\
(\mathrm{mag})\end{array}$ & $\begin{array}{c}\Delta_{((0,1)-(0))} \\
(\mathrm{mag})\end{array}$ & $\begin{array}{r}\text { TRIAD } \\
\text { (mag) }\end{array}$ \\
\hline \multicolumn{9}{|l|}{40 Harmonia } \\
\hline $\bar{\alpha}=4.25$ & $\bar{\alpha}=12.01$ & $\beta_{\mathrm{m}}=0.029$ & & & & & & \\
\hline 0.31 & 1. 21 & 0.004 & & & & & & \\
\hline y 7.402 & $y 7.705$ & $y_{(1,0)} 7.369$ & $V_{(1,0)} 7.369$ & $Q_{y} 0.145$ & $y_{(0)} 7.043$ & $V_{(0)} 7.043$ & $\Delta V \quad 0.33$ & $V_{(0)} 7.14$ \\
\hline 0.050 & 0.060 & 0.048 & 0.048 & 0.021 & 0.045 & 0.045 & 0.09 & \\
\hline b 7.939 & b 8.239 & $b_{(1,0)} 7.903$ & $B_{(1,0)} 8.231$ & $Q_{b} 0.148$ & $b_{(0)} 7.581$ & $B_{(0)} 7.910$ & $\Delta B 0.32$ & \\
\hline 0.050 & 0.061 & 0.048 & 0.059 & 0.021 & 0.045 & 0.195 & 0.25 & \\
\hline$v 8.683$ & $v 8.984$ & $v_{(1,0)} 8.648$ & $U_{(1,0)} 8.629$ & $Q_{v} 0.148$ & $v_{(0)} 8.325$ & $U_{(0)} 8.292$ & $\Delta U 0.34$ & \\
\hline 0.050 & 0.061 & 0.048 & 0.074 & 0.021 & 0.045 & 0.265 & 0.35 & \\
\hline u 9.831 & $u 10.146$ & $u_{(1,0)} 9.810$ & & $Q_{u} 0.122$ & $u_{(0)} 9.460$ & & & \\
\hline 0.061 & 0.065 & 0.051 & & 0.022 & 0.051 & & & \\
\hline$b-y 0.536$ & $b-y 0.535$ & & $B-V 0.862$ & & $b-y 0.538$ & $B-V 0.867$ & $\Delta_{B-V}-0.005$ & $B-V 0.85$ \\
\hline 0.007 & 0.007 & & 0.011 & & 0.090 & 0.150 & & \\
\hline$u-b 1.892$ & $u-b 1.902$ & & $U-B 0.398$ & $Q_{m} 0.141$ & $u-b 1.879$ & $U-B 0.382$ & $\Delta_{U-B} 0.016$ & $U-B 0.43$ \\
\hline 0.021 & 0.020 & & 0.015 & 0.021 & 0.096 & 0.070 & & \\
\hline \multicolumn{9}{|l|}{45 Eugenia } \\
\hline $\bar{\alpha}=1.46$ & $\bar{\alpha}=9.83$ & $\beta_{\mathrm{m}} 0.030$ & & & & & & \\
\hline 0.14 & 0.92 & 0.010 & & & & & & \\
\hline$y 7.661$ & $y 8.098$ & $y_{(1,0)} 7.815$ & $V_{(1,0)} 7.815$ & $Q_{y} 0.142$ & $y_{(0)} 7.504$ & $V_{(0)} 7.504$ & $\Delta V \quad 0.31$ & $V_{(0)} 7.27$ \\
\hline 0.043 & 0.054 & 0.052 & 0.052 & 0.020 & 0.042 & 0.042 & 0.09 & \\
\hline$b 8.086$ & $b 8.216$ & $b_{(1,0)} 8.216$ & $B_{(1,0)} 8.455$ & $Q_{b} 0.174$ & $b_{(0)} 7.935$ & $B_{(0)} 8.193$ & $\Delta B 0.26$ & \\
\hline 0.046 & 0.044 & 0.044 & 0.120 & 0.020 & 0.039 & 0.177 & 0.30 & \\
\hline$v 8.688$ & $v 9.112$ & $v_{(1,0)} 8.828$ & $U_{(1,0)} 8.665$ & $Q_{v} 0.157$ & $v_{(0)} 8.532$ & $U_{(0)} 8.394$ & $\Delta U \quad 0.27$ & \\
\hline 0.049 & 0.051 & 0.044 & 0.161 & 0.019 & 0.039 & 0.279 & 0.44 & \\
\hline$u 9.721$ & u 10.142 & $u_{(1,0)} 9.856$ & & $Q_{u} 0.162$ & $u_{(0)} 9.5650$ & & & \\
\hline 0.082 & 0.041 & 0.066 & & 0.031 & 0.063 & & & \\
\hline$b-y 0.425$ & $b-y 0.401$ & & $B-V 0.659$ & & $b-y 0.431$ & $B-V 0.689$ & $\Delta B-V-0.030$ & $B-V 0.66$ \\
\hline 0.020 & 0.041 & & 0.051 & & 0.081 & 0.135 & & \\
\hline$u-b 1.634$ & $u-b 1.643$ & & $U-B 0.207$ & $Q_{m} 0.159$ & $u-b 1.630$ & $U-B 0.201$ & $\Delta U-B 0.006$ & $U-B 0.27$ \\
\hline 0.055 & 0.056 & & 0.041 & 0.023 & 0.102 & 0.074 & & \\
\hline \multicolumn{9}{|l|}{52 Europa } \\
\hline $\bar{\alpha}=2.77$ & $\bar{\alpha}=7.75$ & $\beta_{\mathrm{m}} 0.040$ & & & & & & \\
\hline 0.21 & 0.81 & 0.012 & & & & & & \\
\hline$y 6.665$ & $y 6.935$ & $y_{(1,0)} 6.640$ & $V_{(1,0)} 6.640$ & $Q_{y} 0.093$ & $y_{(0)} 6.382$ & $V_{(0)} 6.382$ & $\Delta V \quad 0.26$ & $V_{(0)} 6.25$ \\
\hline 0.058 & 0.060 & 0.052 & 0.052 & 0.026 & 0.071 & 0.071 & 0.12 & \\
\hline b 7.094 & b 7.348 & $b_{(1,0)} 7.053$ & $B_{(1,0)} 7.313$ & $Q_{b} 0.127$ & $b_{(0)} 6.822$ & $B_{(0)} 7.086$ & $\Delta B 0.23$ & \\
\hline 0.059 & 0.061 & 0.053 & 0.081 & 0.035 & 0.072 & 0.214 & 0.29 & \\
\hline$v 7.732$ & $v 7.980$ & $v_{(1,0)} 7.685$ & $U_{(1,0)} 7.580$ & $Q_{v} 0.138$ & $v_{(0)} 7.463$ & $U_{(0)} 7.359$ & $\Delta U 0.22$ & \\
\hline 0.058 & 0.063 & 0.054 & 0.107 & 0.035 & 0.073 & 0.335 & 0.44 & \\
\hline$u 8.819$ & u 9.064 & $u_{(1,0)} 8.769$ & & $Q_{u} 0.143$ & $u_{(0)} 8.551$ & & & \\
\hline 0.088 & 0.066 & 0.061 & & 0.046 & 0.094 & & & \\
\hline$b-y 0.429$ & $b-y 0.413$ & & $B-V 0.673$ & & $b-y 0.440$ & $B-V 0.704$ & $\Delta B-V-0.031$ & $B-V 0.66$ \\
\hline 0.021 & 0.014 & & 0.029 & & 0.143 & 0.239 & & \\
\hline$u-b 1.725$ & $u-b 1.717$ & & $U-B 0.267$ & $Q_{m} 0.125$ & $u-b 1.729$ & $U-B 0.273$ & $\Delta U-B-0.006$ & $U-B 0.33$ \\
\hline 0.062 & 0.036 & & 0.036 & 0.036 & 0.166 & 0.121 & & \\
\hline
\end{tabular}

also shown in Table 2, together with the differences found between the Johnson magnitudes calculated using a linear phase correction, $\overline{U B V}(1,0)$, and those obtained by using the Lumme and Bowell relation $\overline{U B V}(0)$, (values $\Delta_{((0,1)-(0))}$ in Table 2).

In order to obtain the corresponding rotational synodic periods for each asteroid, analysis of frequencies were carried out on our data using the method described in Rodríguez et al. (1998). Synodic periods of 0.37125 , 0.23750 and 0.23456 are obtained for 40 Harmonia, 45 Eugenia and 52 Europa, respectively.

Figures 1 to 3 show the composite lightcurves derived using these synodic periods for each asteroid. The $(1,0)$ magnitudes derived in the different Strömgren filters and the $b-y, v-b$ and $u-b$ colour indices versus the rotational phase are also shown in these figures. The Strömgren 


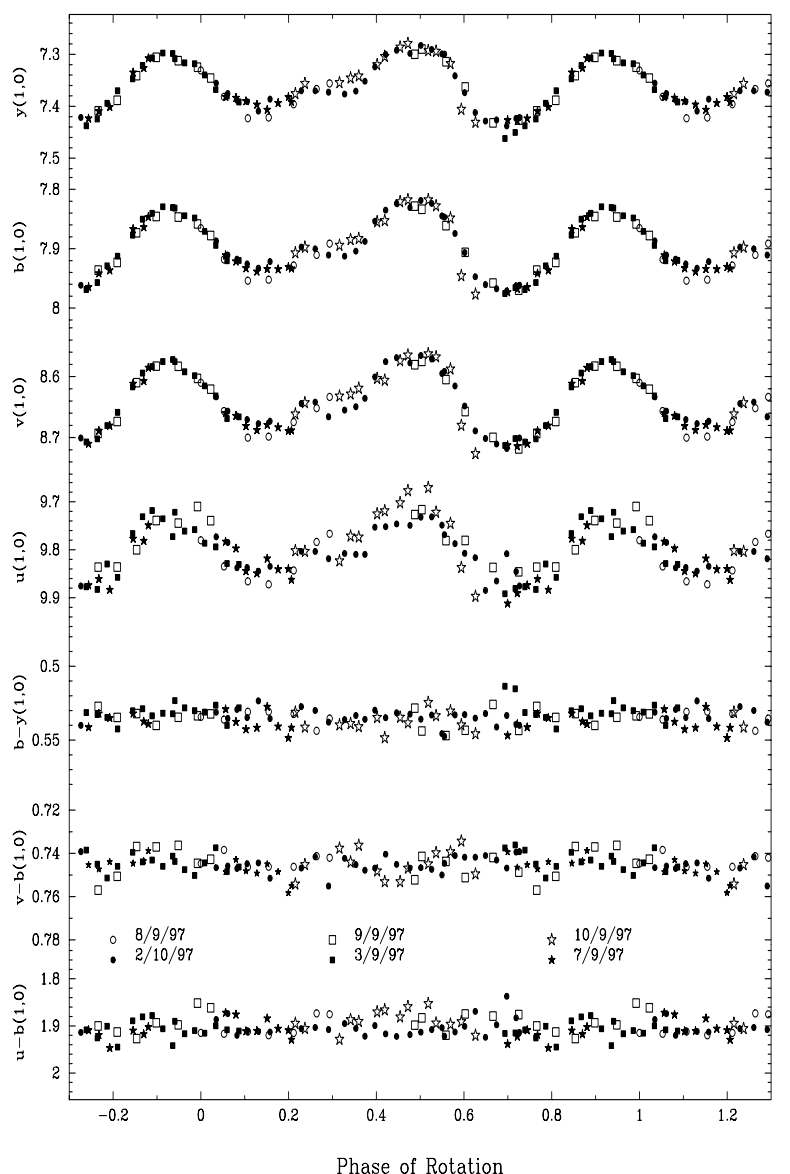

Fig. 1. Lightcurves and colour indices of 40 Harmonia in rotational phase. The 0 phase time corresponds at JD 2450700.5388 corrected for light-time

magnitudes observed during September need a greater phase angle correction than that applied to those observed during October when the phase angles are greater than $7^{\circ}$. Therefore September magnitudes are shifted by additional constants of $+0^{\mathrm{m}} 07,+0.18$ and $+0{ }^{\mathrm{m}} 05$, in all the uvby filters, for 40 Harmonia, 45 Eugenia and 52 Europa, respectively. The composite lightcurves obtained for each asteroid show very regular shapes with two maxima and two minima per rotation cycle.

\subsection{Poles and shapes}

Poles and shapes for these asteroids have been determined using the Epoch/Amplitude method (see Taylor 1979; Magnusson 1986 and Magnusson et al. 1989). Lightcurve data reported in the literature together with lightcurves obtained here for these asteroids have been used in the analysis. Most of the lightcurves used in this work can be found in the Asteroid Photometric Catalogue by Lagerkvist et al. (1987, 1988, 1992). The

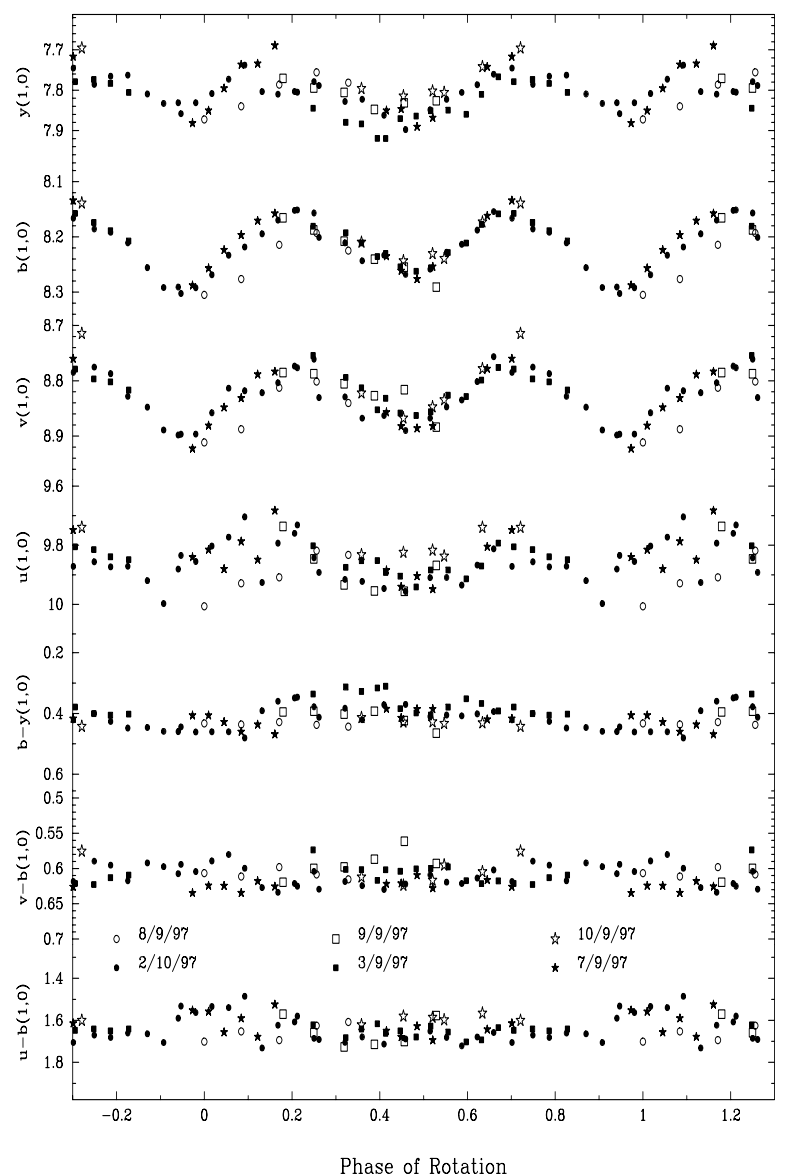

Fig. 2. Lightcurves and colour indices of 45 Eugenia in rotational phase. The 0 phase time corresponds at JD 2450700.5473 corrected for light-time

epochs of maximum brightness and the lightcurve amplitudes used for each asteroid together with the references for the lightcurves used are listed in Table 3.

We use the method proposed by Michalowski \& Velichko (1990) that use the Epoch and Amplitude equations to build expressions, $f_{\mathrm{l}}\left(\right.$ T sid, $\left.\lambda_{\mathrm{p}}, \beta_{\mathrm{p}}, b / a, b / c, \beta_{\mathrm{A}}\right)=$ 0 for $l=1, \ldots(k+m)$, where $k$ is the number of pairs of lightcurves used for the Epoch equations, $m$ is the number of lightcurves used for the Amplitude equations, Tsid is the sidereal period of rotation of the asteroid, $\lambda_{\mathrm{p}}$ and $\beta_{\mathrm{p}}$ are the pole coordinates of the asteroid, $a \geq b \geq c$ are the symmetry axes of the asteroid (considered as a triaxial ellipsoid of axis $a \geq b \geq c$ rotating about their shortest axis) and $\beta_{\mathrm{A}}$ is a phase coefficient taking into account the phase angle effects on the amplitude of the lightcurves. Following the work of De Angelis (1993), we have used the same procedure of standardization of the variables $f_{1}$, dividing each $f_{1}$ by the standard deviation $\sigma$ of all $f_{1}$ of the same group of equations, and then, these 
Table 3. Epochs of maximum brightness and Amplitudes observed

\begin{tabular}{|c|c|c|c|c|c|c|}
\hline Date & JD & $\begin{array}{c}\text { Ecliptic } \\
\text { Long } \\
(1950)\end{array}$ & $\begin{array}{r}\text { Ecliptic } \\
\text { Lat } \\
(1950)\end{array}$ & $\begin{array}{l}\text { Phase } \\
\text { (deg) }\end{array}$ & $\begin{array}{l}\text { Amp. } \\
\text { (mag) }\end{array}$ & Reference \\
\hline \multicolumn{7}{|l|}{40 Harmonia } \\
\hline 1411958 & 2436217.8864 & 146.93 & 4.37 & 13.48 & - & Gehrels and Owings (1962) \\
\hline 2911958 & 2436232.7303 & 143.89 & 4.99 & 6.65 & 0.23 & Gehrels and Owings (1962) \\
\hline 2911958 & 2436232.9298 & 143.84 & 5.00 & 6.55 & - & Gehrels and Owings (1962) \\
\hline 1421975 & 2442457.5334 & 127.61 & 4.73 & 7.25 & 0.28 & Lagerkvist (1978) \\
\hline 6101983 & 2445614.2994 & 291.30 & -3.62 & 26.81 & 0.30 & McCheyne et al. (1985) \\
\hline 7101983 & 2445615.3196 & 291.56 & -3.62 & 26.86 & - & McCheyne et al. (1985) \\
\hline 8101983 & 2445616.3273 & 291.82 & -3.61 & 26.91 & - & McCheyne et al. (1985) \\
\hline $8 \quad 51986$ & 2446558.7162 & 233.41 & 5.12 & 3.59 & 0.15 & Gallardo and Tancredi (1987) \\
\hline 991997 & 2450701.4624 & 351.62 & -7.68 & 4.24 & - & This work \\
\hline 1091997 & 2450701.6148 & 351.58 & -7.68 & 4.20 & 0.15 & This work \\
\hline 1191997 & 2450702.5703 & 351.34 & -7.70 & 3.95 & - & This work \\
\hline 2101997 & 2450724.4850 & 346.17 & -7.56 & 10.90 & 0.15 & This work \\
\hline 3101997 & 2450725.3888 & 346.00 & -7.54 & 11.33 & - & This work \\
\hline \multicolumn{7}{|l|}{45 Eugenia } \\
\hline 1161969 & 2440383.6909 & 217.87 & 9.96 & 16.05 & - & Taylor et al. (1988) \\
\hline $4 \quad 51978$ & 2443632.6831 & 226.59 & 10.77 & 4.56 & 0.29 & Debehogne and Zappala (1980) \\
\hline 161978 & 2443660.6991 & 221.04 & 10.50 & 11.95 & - & Harris and Young (1979) \\
\hline 1311982 & 2444982.9384 & 172.49 & 0.95 & 18.66 & 0.17 & Weidenschilling et al. (1987) \\
\hline $13 \quad 31982$ & 2445041.5846 & 164.76 & 3.62 & 2.97 & - & Debehogne et al. (1983) \\
\hline 2151982 & 2445110.6812 & 161.27 & 4.68 & 22.75 & 0.20 & Weidenschilling et al. (1987) \\
\hline 1471982 & & 176.10 & 4.60 & 21.40 & 0.18 & Weidenschilling et al. (1987) \\
\hline 2151983 & 2445476.0576 & 298.15 & 6.82 & 19.90 & 0.14 & Weidenschilling et al. (1987) \\
\hline $30 \quad 61983$ & 2445515.9700 & 294.80 & 6.81 & 7.17 & 0.11 & Weidenschilling et al. (1987) \\
\hline 11101983 & 2445618.7777 & 290.34 & 1.91 & 22.04 & 0.15 & Weidenschilling et al. (1987) \\
\hline 11111983 & 2445649.6401 & 298.90 & 0.89 & 20.45 & 0.15 & Weidenschilling et al. (1987) \\
\hline 2991984 & 2445972.7803 & 35.09 & -8.14 & 9.97 & - & Taylor et al. (1988) \\
\hline 24101984 & 2445998.1970 & 29.82 & -8.87 & 3.03 & - & Taylor et al. (1988) \\
\hline 18111984 & & 24.90 & -8.80 & 10.2 & 0.36 & Weidenschilling et al. (1987) \\
\hline 27111984 & 2446032.1503 & 23.74 & -8.52 & 13.01 & - & Taylor et al. (1988) \\
\hline 1711985 & 2446082.7266 & 26.52 & -6.86 & 19.51 & 0.41 & Weidenschilling et al. (1987) \\
\hline 20101985 & 2446359.0875 & 119.07 & -5.22 & 20.27 & 0.15 & Weidenschilling et al. (1987) \\
\hline 1711986 & 2446447.8954 & 116.55 & -5.23 & 1.83 & 0.09 & Weidenschilling et al. (1987) \\
\hline $16 \quad 61987$ & 2446962.6469 & 223.69 & 10.06 & 15.59 & - & Lebofsky et al. (1988) \\
\hline 991997 & 2450700.6000 & 348.34 & -3.57 & 1.58 & 0.12 & This work \\
\hline 2101997 & 2450724.4542 & 343.44 & -4.21 & 8.97 & 0.12 & This work \\
\hline 3101997 & 2450725.4125 & 343.28 & -4.22 & 9.32 & - & This work \\
\hline \multicolumn{7}{|l|}{52 Europa } \\
\hline 17111976 & 2443100.3604 & 64.73 & -10.51 & 4.88 & - & Scaltriti and Zappala (1977) \\
\hline 18111976 & 2443100.6041 & 64.68 & -10.51 & 4.82 & - & Scaltriti and Zappala (1977) \\
\hline 19111976 & 2443102.4801 & 64.29 & -10.50 & 4.35 & - & Scaltriti and Zappala (1977) \\
\hline 11121976 & 2443124.2884 & 59.81 & -9.93 & 7.52 & 0.09 & Scaltriti and Zappala (1977) \\
\hline 12121976 & 2443124.5264 & 59.76 & -9.92 & 7.60 & - & Scaltriti and Zappala (1977) \\
\hline $24 \quad 11983$ & 2445359.3366 & 119.91 & -1.52 & 1.49 & 0.10 & Zappala et al. (1983) \\
\hline 2411983 & 2445359.4646 & 119.91 & -1.52 & 1.49 & - & Zappala et al. (1983) \\
\hline $25 \quad 11983$ & 2445359.5729 & 119.86 & -1.51 & 1.58 & - & Zappala et al. (1983) \\
\hline $25 \quad 41984$ & 2445815.5260 & 211.19 & 10.83 & 3.58 & 0.08 & Barucci et al. (1986) \\
\hline 291986 & 2446675.5158 & 347.15 & -6.32 & 3.26 & 0.23 & Dotto et al. (1995) \\
\hline 291986 & 2446675.6309 & 347.12 & -6.33 & 3.22 & - & Dotto et al. (1995) \\
\hline 2111992 & 2448928.7170 & 26.44 & -10.90 & 5.53 & 0.10 & Michalowski et al. (1995) \\
\hline 821994 & 2449391.7604 & 124.43 & 0.16 & 4.94 & 0.11 & Michalowski et al. (1995) \\
\hline 821994 & 2449391.8824 & 124.41 & 0.16 & 4.99 & - & Michalowski et al. (1995) \\
\hline 991997 & 2450700.6467 & 351.99 & -7.22 & 2.95 & 0.20 & This work \\
\hline 1191997 & 2450702.5240 & 351.62 & -7.27 & 2.57 & - & This work \\
\hline 2101997 & 2450724.3380 & 347.39 & -7.61 & 6.95 & 0.20 & This work \\
\hline 3101997 & 2450725.3984 & 347.21 & -7.61 & 7.29 & - & This work \\
\hline 7101997 & 2450729.3837 & 346.58 & -7.61 & 8.52 & - & This work \\
\hline
\end{tabular}




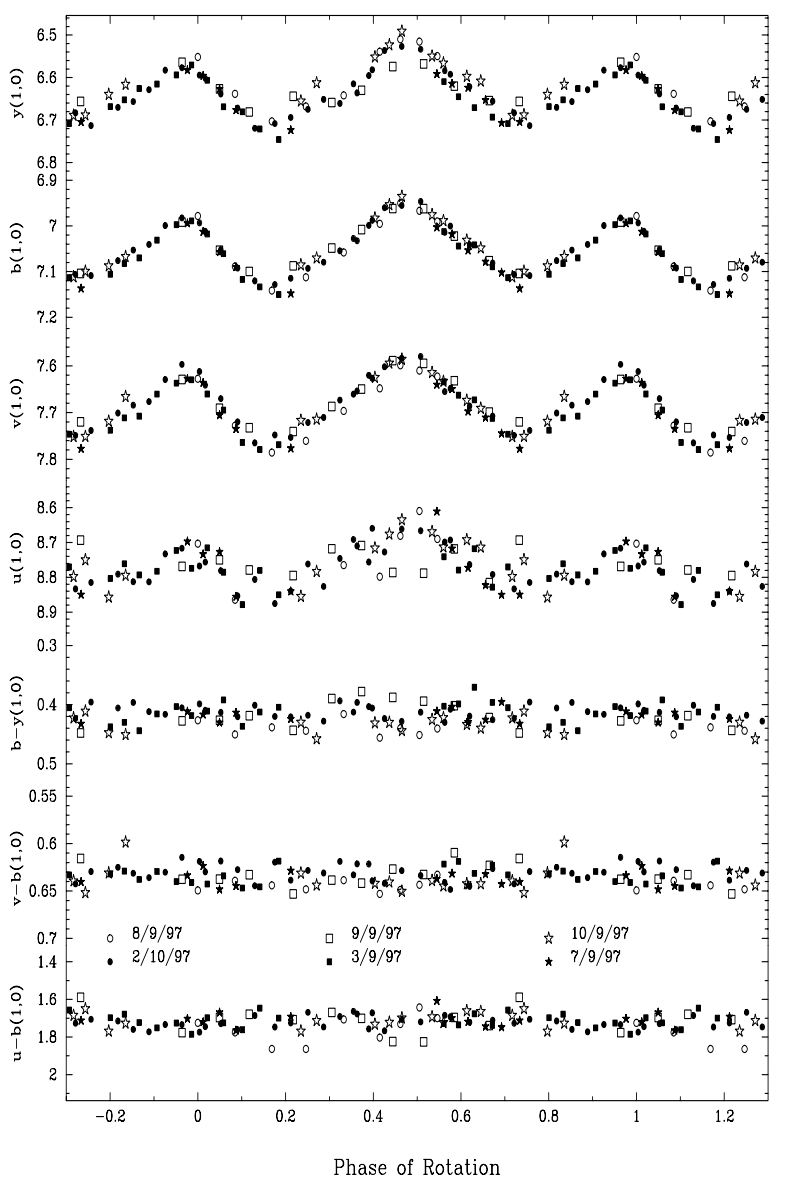

Fig. 3. Lightcurves and colour indices of 52 Europa in rotational phase. The 0 phase time corresponds at JD 2450700.5384 corrected for light-time

expressions for $f_{1}$ have been used as elements of a sum of squares to be minimized by a least-square fit.

First of all we look for a mean synodic period that suits all the epochs of maximum light for each asteroid. There are cases where this mean synodic period is not well defined, as it is possible to choose between different mean synodic periods. For these cases we look for different solutions for each of the possible mean synodic periods by using a grid of $\lambda_{\mathrm{p}}$ and $\beta_{\mathrm{p}}$ as trial poles making a leastsquare fit for each trial pole, first for prograde and then for retrograde solutions. The solutions with least residuals are considered as the most probable solutions. In this way we obtain a more likely solution for the corresponding values of $T$ sid, $\lambda_{\mathrm{p}}, \beta_{\mathrm{p}}, a / b, b / c$ and $\beta_{\mathrm{A}}$ simultaneously.

In Table 4 we have listed the final solution obtained for $T$ sid, $\lambda_{\mathrm{p}}, \beta_{\mathrm{p}}, a / b, b / c$ and $\beta_{\mathrm{A}}$ for each asteroid, together with the mean synodic period used. Previous solutions reported by earlier authors are also shown in Table 4 .

\section{Results}

\subsection{Harmonia}

40 Harmonia is a S-type asteroid (Tholen 1989) with a diameter of $111 \mathrm{~km}$ (Tedesco 1989). From our data we find a synodic period for 40 Harmonia of $8^{\mathrm{h}} 54^{\mathrm{m}} 36^{\mathrm{s}}$. The composite lightcurves obtained for 40 Harmonia show a regular shape in all the uvby filters, with two maxima and two minima per rotational cycle. The maximum amplitude in all the uvby filters is of 0.15 and the amplitude averaged in the rotational cycle is of 0.13 . No significative difference in the lightcurve amplitude is found in the lightcurves measured during October, $\bar{\alpha}=12.001$, with respect to those measured during September, $\bar{\alpha}=4^{\circ} .025$. The colour indices do not show any variation greater than the scatter of the data during the rotational phase of this asteroid (see Fig. 1).

Using a linear phase angle correction, a mean linear phase coefficient, $\beta_{\mathrm{m}}$, of $0.029 \pm 0.004 \mathrm{mag} /$ degree is obtained by averaging the linear phase coefficients obtained in each of the Strömgren filters. The mean values of $V(1,0)=7^{\mathrm{m}} 369, B-V=0.862$ and $U-B=0.398$ found using a linear phase angle correction, agree with the mean values of $V(0)=7^{\mathrm{m}} 043, B-V=0.867$ and $U-B=0.382$ found by using the radiative transfer theory of Lumme \& Bowell (1981b) and with the values reported in the TRIAD file of Bowell et al. (1979). The difference found in the magnitudes deduced for both methods, $V(1,0)-V\left(0^{\circ}\right)$, of 0.33 is as expected for all the asteroids (Bowell \& Lumme 1979). The multiple scattering factor obtained from the $u$ Strömgren filter, $Q_{u}$, is smaller than that obtained from the other Strömgren filters, that have very similar values. However the difference found for the $Q_{u}$ factor is within the error bars of the determination and thus may not be significant. The average value found in all the Strömgren filters of the multiple scattering factor, $Q_{m}$, of $0.141 \pm 0.021$ is in agreement with the mean value of an asteroid of type S (Bowell \& Lumme 1979).

Tancredi \& Gallardo (1991) determined for this asteroid values of $\lambda_{\mathrm{p}}$ of $15^{\circ}-25^{\circ}$ and $\beta_{\mathrm{p}}$ of $20^{\circ}-60^{\circ}$ (or $\left.\lambda_{\mathrm{p}}=195^{\circ}-210^{\circ}, \beta_{\mathrm{p}}=20^{\circ}-70^{\circ}\right)$ and a value for $a / b$ of $1.27-1.35$. Michalowski (1993) obtained a prograde sense of rotation with a $T$ sid $=0.3712522$ and values $\lambda_{\mathrm{p}}=208^{\circ}$, $\beta_{\mathrm{p}}=21^{\circ}, a / b=1.27$ and $b / c=2.07$.

Here we obtain the best fit considering 40 Harmonia as a prograde rotator obtaining Tsid $=0.3711872$ being $\lambda_{\mathrm{p}}=22^{\circ}, \beta_{\mathrm{p}}=28^{\circ}\left(\right.$ or $\left.\lambda_{\mathrm{p}}=203^{\circ}, \beta_{\mathrm{p}}=38^{\circ}\right), a / b=1.31$, $b / c=1$ and $\beta_{\mathrm{A}}=110^{-5}$. A solution with a value of Tsid $=0.3712535$ is also obtained but this solution has slightly greater residuals.

The observed amplitudes together with the theoretical amplitudes, at zero-phase angle, obtained with the solution values of $a / b$ and $b / c$ versus the aspect angle are plotted in Fig. 4. The agreement obtained is surprisingly good, however more lightcurves of 40 Harmonia would improve 
Table 4. Rotational properties

\begin{tabular}{|c|c|c|c|c|c|c|c|c|c|c|}
\hline Reference & Mean Tsyn & Sense & Tsid & $\lambda_{\mathrm{p}}$ & $\beta_{\mathrm{p}}$ & $\lambda_{\mathrm{p}}$ & $\beta_{\mathrm{p}}$ & $a / b$ & $b / c$ & $\beta_{\mathrm{A}}$ \\
\hline \multicolumn{11}{|l|}{40 Harmonia } \\
\hline Tancredi \& Gallardo (1991) & & & & $20^{\circ}$ & $40^{\circ}$ & $203^{\circ}$ & $45^{\circ}$ & 1.31 & 1 & \\
\hline Michalowski (1993) & & $\mathrm{P}$ & 0.3712522 & & & $208^{\circ}$ & $21^{\circ}$ & 1.24 & 2.07 & \\
\hline This work & 0. 3712973 & $\mathrm{P}$ & 0.3711872 & $22^{\circ}$ & $28^{\circ}$ & $203^{\circ}$ & $38^{\circ}$ & 1.31 & 1 & 0.00001 \\
\hline This work & 0.3713638 & $\mathrm{P}$ & 0.3712535 & $12^{\circ}$ & $34^{\circ}$ & $201^{\circ}$ & $41^{\circ}$ & 1.31 & 1 & 0.00001 \\
\hline \multicolumn{11}{|l|}{45 Eugenia } \\
\hline Taylor et al. (1988) & & $\mathrm{R}$ & 0.2374645 & $106^{\circ}$ & $26^{\circ}$ & $295^{\circ}$ & $34^{\circ}$ & & & \\
\hline Drummond et al. (1988) & & $\mathrm{R}$ & 0.2374646 & & & $307^{\circ}$ & $44^{\circ}$ & 1.33 & 1.65 & \\
\hline Magnusson (1990) & & $\mathrm{R}$ & 0.2374646 & $116^{\circ}$ & $26^{\circ}$ & $305^{\circ}$ & $35^{\circ}$ & 1.36 & 1.48 & \\
\hline Lumme et al. (1990) & & $\mathrm{R}$ & 0.2374646 & $128^{\circ}$ & $16^{\circ}$ & $313^{\circ}$ & $25^{\circ}$ & 1.3 & 1.4 & \\
\hline Drummond et al. (1991) & & $\mathrm{R}$ & 0.2374646 & & & $307^{\circ}$ & $44^{\circ}$ & 1.33 & 1.65 & \\
\hline De Angelis (1995) & & $\mathrm{R}$ & 0.2374650 & & & $289^{\circ}$ & $27^{\circ}$ & 1.33 & 1.23 & 0.0054 \\
\hline This work & 0. 2374297 & $\mathrm{R}$ & 0.2374644 & $106^{\circ}$ & $42^{\circ}$ & $313^{\circ}$ & $41^{\circ}$ & 1.33 & 1.4 & 0.0030 \\
\hline \multicolumn{11}{|l|}{52 Europa } \\
\hline Barucci et al. (1986) & & & & $0^{\circ}$ & $37^{\circ}$ & $203^{\circ}$ & $38^{\circ}$ & 1.12 & 1 & \\
\hline Dotto et al. (1995) & & $\mathrm{R}$ & 0.2346504 & $70^{\circ}$ & $40^{\circ}$ & $260^{\circ}$ & $55^{\circ}$ & 1.21 & 1.30 & \\
\hline Michalowski et al. (1995) & & $\mathrm{R}$ & 0.2347019 & $77^{\circ}$ & $18^{\circ}$ & $264^{\circ}$ & $32^{\circ}$ & 1.20 & 1.17 & \\
\hline This work & 0.2346131 & $\mathrm{P}$ & 0.2345855 & $63^{\circ}$ & $46^{\circ}$ & $261^{\circ}$ & $60^{\circ}$ & 1.19 & 2.2 & 0.0050 \\
\hline
\end{tabular}

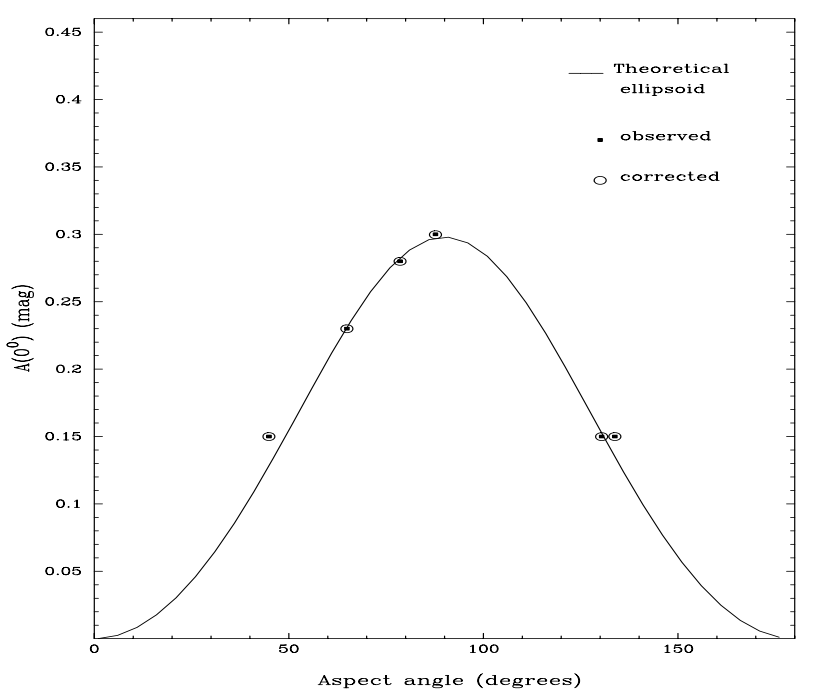

Fig. 4. Amplitude obtained considering 40 Harmonia as a triaxial ellipsoid with $a / b=1.31$ and $b / c=1$. Amplitudes observed and corrected by a phase factor $\beta_{\mathrm{A}}=0.00001$

the determination of its sidereal period and rotational parameters.

\subsection{Eugenia}

45 Eugenia is a $214 \mathrm{~km}$ FC-type asteroid (Tedesco 1989; Tholen 1989). This asteroid has been observed during eight oppositions between 1969 and 1988 and now in 1997 . We find a synodic period of $5^{\mathrm{h}} 42^{\mathrm{m}} 00^{\mathrm{s}}$ from our data. The composite lightcurves obtained for 45 Eugenia show regular shapes in all the uvby filters, with two maxima and two minima per rotational cycle. The scatter of the data is greater in the $u$ and $y$ filters. The maximum amplitude in all the uvby filters is of 0.12 and the amplitude averaged in the rotational cycle is of 0.11 . No significant differences in the amplitude can be deduced from our data for October observations, $\bar{\alpha}=9.83$, with respect to September observations, $\bar{\alpha}=1$.046. The colour indices during the rotational phase of this asteroid show large dispersions. The $b-y$ colour index seems to present maximum values at half of the rotational phase of this asteroid while the $u-b$ colour index seems to show a variation anticorrelated with that of the $b-y$ curve. However these variations are within the scatter of the data and may be of no significance (see Fig. 2).

Using a linear phase angle correction a mean linear phase coefficient of $0.030 \pm 0.010 \mathrm{mag} /$ degree is obtained from all the Strömgren filters. The mean values of $V(1,0)=7 \mathrm{~m} 815, B-V=0.659$ and $U-B=0.9207$ found using a linear phase angle correction and the ones of $V(0)=7{ }^{\mathrm{m}} 504, B-V=0.689$ and $U-B=0.201$ found following Lumme \& Bowell (1981b) theory are in very good agreement. However, the value of $U-B$ obtained here is smaller than the one reported by the TRIAD file. The multiple scattering factors, $Q_{\text {filters }}$, obtained from the different Strömgren filters do not vary greatly. The average value found in all the Strömgren filters, $Q_{m}$, is of $0.159 \pm 0.023$. These values are greater than the mean 


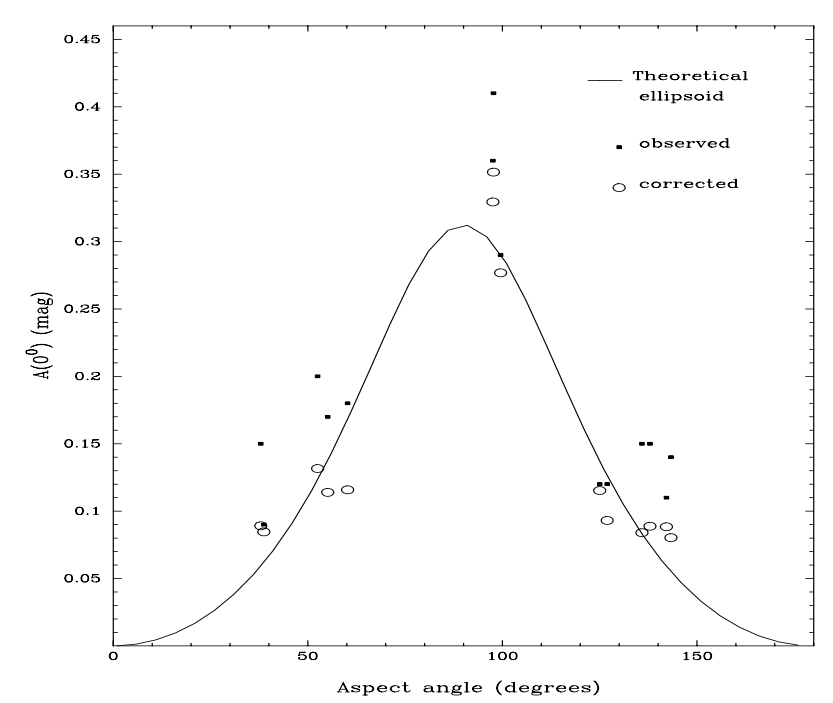

Fig. 5. Amplitude obtained considering 45 Eugenia as a triaxial ellipsoid with $a / b=1.33$ and $b / c=1.4$. Amplitudes observed and corrected by a phase factor $\beta_{\mathrm{A}}=0.003$

values reported for $\mathrm{C}$ type asteroids, and also for $\mathrm{U}$ type asteroids (Bowell \& Lumme 1979; Lumme \& Bowell 1981b).

Previous solutions show 45 Eugenia as a retrograde rotator. Here we obtain the best fit with $T$ sid $=0$ d 2374644 and $\lambda_{\mathrm{p}}=313^{\circ}$ and $\beta_{\mathrm{p}}=41^{\circ}$ or $\left(\lambda_{\mathrm{p}}=106^{\circ}\right.$ and $\left.\beta_{\mathrm{p}}=42^{\circ}\right)$ and values for $a / b$ of $1.33, b / c$ of 1.4 and $\beta_{\mathrm{A}}$ of $310^{-3}$. This solution is in agreement with previous determined values for $\lambda_{\mathrm{p}}$ and $a / b$, however the value of $\beta_{\mathrm{p}}$ seems to be greater than the average value of the previous determinations (although Drummond et al. 1988, 1991, reported values of $\left.\beta_{\mathrm{p}}=44^{\circ}\right)$.

In Fig. 5 the observed amplitudes together with the theoretical, at zero phase angle, obtained with these values of $a / b$ and $b / c$ are plotted versus the aspect angle. This figure shows very dispersed amplitude values, for aspect angles from $40^{\circ}$ to $60^{\circ}$, from $120^{\circ}$ to $150^{\circ}$ and close to $100^{\circ}$. More lightcurves covering the gaps in the aspect angle would help us to discern the rotational parameters of 45 Eugenia.

\subsection{Europa}

This object is a CF-type asteroid (Tholen 1989) with a diameter of $312 \mathrm{~km}$ (Tedesco 1989). Zappala et al. (1983) observed 52 Europa in January 1983 and obtained a synodic period of 5.631 and an amplitude of 0.10 . From our data we find a synodic period of $5^{\mathrm{h}} 37^{\mathrm{m}} 46^{\mathrm{s}}$. The composite lightcurves obtained for 52 Europa show regular shapes in all the uvby filters, with two maxima and two minima per rotational cycle (see Fig. 3). The maximum amplitude in all the uvby filters is of 0.20 and the amplitude averaged in the rotational cycle is of 0.14 . No significant differences in the lightcurve amplitude can be deduced from our data at $\bar{\alpha}=7.75$ (October observations) with respect to those at $\bar{\alpha}=2.77$ (September observations) and the colour indices seem to be constant during the rotational phase of the asteroid.

A mean linear phase coefficient, $\beta_{\mathrm{m}}$, of $0.040 \pm$ $0.012 \mathrm{mag} /$ degree is obtained from all the Strömgren filters. This coefficient is obtained for an average phase angle of 7.75 when a possible greater phase correction than the one deduced at greater phase angles is to be expected. We find mean values of $V(1,0)=6^{\mathrm{m}} 640$, $B-V=0.673$ and $U-B=0.267$ using a linear phase angle correction that agree with the mean values of $V(0)=6.382, B-V=0.704$ and $U-B=0.273$ obtained by using Lumme \& Bowell (1981b) theory. The difference $V(1,0)-V(0)=0{ }^{\mathrm{m}} 26$ is slightly smaller than the expected but could be explained by considering that the magnitude, $V(1,0)$, has been obtained with a linear phase correction to values obtained at phase angles between $7^{\circ}$ and $9^{\circ}$ and, as was commented before, the linear phase coefficient used could be a little greater than that obtained at greater phase angles, producing a consecutive decrease in the extrapolation to the $V(1,0)$ magnitude. The values obtained here for the magnitude and the colour indices agree with the values reported in the TRIAD file.

There are slight differences in the values of the multiple scattering factors, $Q_{\text {filters }}$, deduced using the different Strömgren filters. The value obtained in the $y$ filter is the smallest one, increasing for the $b, v$ and $u$ filters. This value, $Q_{y}=0.093 \pm 0.026$, is within the range of the mean values of $Q_{V}$ reported for a C-type asteroids (Bowell \& Lumme 1979).

Scaltriti \& Zappala (1977), Zappala et al. (1983) and Barucci et al. (1986) observed 52 Europa in December 1976, January 1983 and April 1984 finding amplitudes in their lightcurves of $0 .{ }^{\mathrm{m}} 09,0.10$ and $0{ }^{\mathrm{m}} 08$, respectively. Dotto et al. (1995) observed 52 Europa during AugustSeptember 1986 and obtained a lightcurve of 0.23 of amplitude. They found 52 Europa as a retrograde rotator with Tsid $=0.2346504$ and $\lambda_{\mathrm{p}}=70^{\circ}, \beta_{\mathrm{p}}=40^{\circ}$ (or $\left.\lambda_{\mathrm{p}}=260^{\circ}, \beta_{\mathrm{p}}=55^{\circ}\right)$ and values for $a / b$ of 1.21 and for $b / c$ of 1.30. Michalowski et al. (1995) observed 52 Europa in 1992 and 1994 obtaining lightcurves with 0.10 and 0.11 of amplitude, respectively. They obtained 52 Europa as a retrograde rotator with $T$ sid $=0.2347019$ and $\lambda_{\mathrm{p}}=77^{\circ}$, $\beta_{\mathrm{p}}=18^{\circ}\left(\right.$ or $\lambda_{\mathrm{p}}=264^{\circ}, \beta_{\mathrm{p}}=32^{\circ}$ ) and values for $a / b$ of 1.20 and for $b / c$ of 1.17 .

Here we obtain the best fit when 52 Europa is considered as a prograde rotator (when 52 Europa is considered as a retrograde rotator the residuals coming from the Epoch method increase substantially) obtaining Tsid = $0 \mathrm{~d} 2345855$ and $\lambda_{\mathrm{p}}=261^{\circ}, \beta_{\mathrm{p}}=60^{\circ}$ (or $\lambda_{\mathrm{p}}=63^{\circ}$, $\left.\beta_{\mathrm{p}}=46^{\circ}\right)$ and values for $a / b$ of 1.19 , for $b / c$ of 2.2 and for $\beta_{\mathrm{A}}=510^{-3}$. These results are in agreement with 


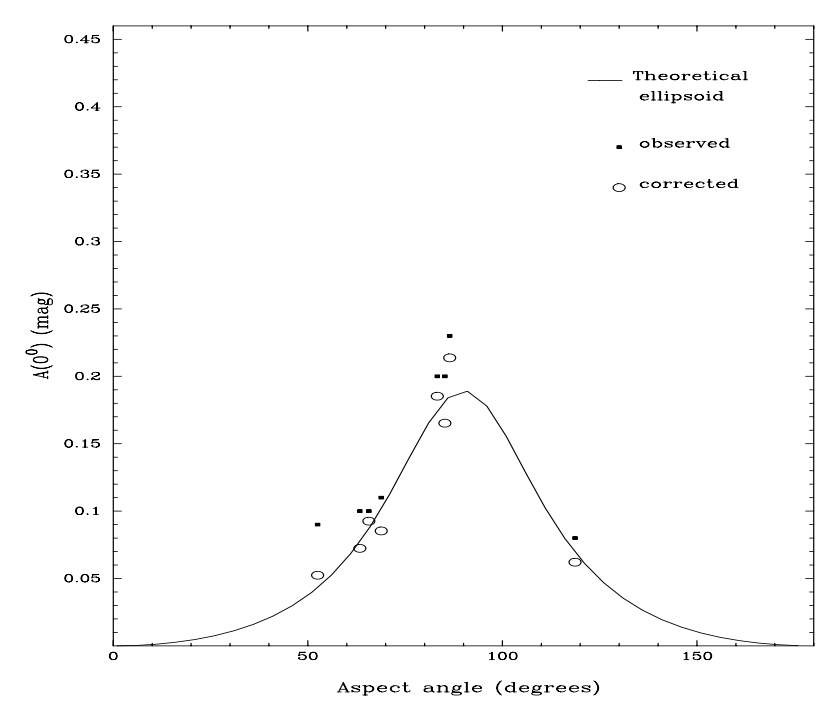

Fig. 6. Amplitude obtained considering 52 Europa as a triaxial ellipsoid with $a / b=1.19$ and $b / c=2.2$. Amplitudes observed and corrected by a phase factor $\beta_{\mathrm{A}}=0.005$

previous determinations for $\lambda_{\mathrm{p}}$ and $\beta_{\mathrm{p}}$ obtained by Dotto et al. (1995). However the values of $\beta_{\mathrm{p}}$ obtained by Michalowski et al. (1995) are smaller. The value obtained for $a / b$ is in agreement with previous determinations while the value found for $b / c$ is greater than previous determinations. The values of the observed amplitudes and the theoretical ones calculated with this solution at zero phase angle are plotted in Fig. 6. The agreement obtained is very good. Again new lightcurves for increasing the ecliptic longitude coverage of this asteroid would help to improve future work on the rotational properties of 52 Europa.

\section{Conclusions}

Lightcurves of asteroids 40 Harmonia, 45 Eugenia and 52 Europa from September to October 1997 are presented using uvby Strömgren photometry. The lightcurves obtained for these three asteroids show very regular shapes with two maxima and two minima per cycle. Although in all the cases the two peaks are of slightly different amplitudes. The absolute magnitudes and colour indices obtained agree well with previous measurements. No significative tendencies are found in the colour indices during their rotational phases. The values for the linear phase coefficients and for the multiple scattering factors obtained for 40 Harmonia and 52 Europa are in good agreement with the mean values expected for S-type and C-type asteroids, respectively. However, for 45 Eugenia a multiple scattering factor greater than the mean values expected for C-type asteroids (also for U-type) is obtained.

Values of sidereal periods, poles and shapes parameter are proposed. However lightcurves taken at different eclip- tic longitudes and at different solar phase angles would help to obtain more accurate rotational and shape solutions.

Acknowledgements. This research was partially supported by the Comisión Interministerial de Ciencia y Tecnología under contracts ESP97-1773-C03-01 and ESP97-1798, the Junta de Andalucía and the Dirección General de Enseñanza Superior (DGES) under project PB96-0840. This research has made use of the Asteroid Photometric Catalogue database. We very gratefully acknowledge the staffs of Sierra Nevada Observatory for their help during the run of observations. Acknowledgements are also especially made to M.C. Romero for making available many papers used in this investigation and to V.G. Brown for proofreading. We wish to thank the referee, J. Berthier, for useful comments and suggestions.

\section{References}

Barucci M.A., Bockelee-Morvan D., Brahic A., et al., 1986, A\&A 163, 261

Bowell E., Lumme K., 1979, in Asteroids I, Gehrels T. (ed.). Univ. of Arizona press, Tucson, p. 132

Bowell E., Gehrels T., Zellner B., 1979, in Asteroids I, Gehrels T. (ed.). Univ. of Arizona press, Tucson, p. 1108

De Angelis G., 1993, PSS 41, 285

De Angelis G., 1995, PSS 43, 649

Debehogne H., Zappala V., 1980, A\&AS 40, 257

Debehogne H., De Sanctis G., Zappala V., 1983, Icarus 55, 236

Dermott S.F., Harris A.W., Murray C.D., 1984, Icarus 57, 14

Dotto E., De Angelis G., Di Martino M., et al., 1995, Icarus 117,313

Drummond J.D., Weidenschilling, S.J., Chapman C.R., Davis D.R., 1988, Icarus 76, 19

Drummond J.D., Weidenschilling, S.J., Chapman C.R., Davis D.R., 1991, Icarus 89, 44

Farinella P., Paolicchi P., Zappala V., 1981, A\&A 104, 159

Gallardo T., Tancredi G., 1987, Rev. Mex. Astron. Astrofis. 15,103

Gehrels T., Owings D., 1962, ApJ 135, 906

Harris A.W., Young J.W., 1979, Icarus 38, 100

Hauck B., Mermilliod M., 1998, A\&AS 129, 431

Lagerkvist C.I., 1978, A\&AS 31, 361

Lagerkvist C.I., Barucci M.A., Capria M.T., et al., 1987, Asteroid Photometric Catalogue, Consiglio Nazionale delle Richerche, Roma

Lagerkvist C.I., Barucci M.A., Capria M.T., et al., 1988, Asteroid Photometric Catalogue, First Update. Consiglio Nazionale delle Richerche, Roma

Lagerkvist C.I., Barucci M.A., Capria M.T., et al., 1992, Asteroid Photometric Catalogue, Second Update. Uppsala Universitet, Uppsala

Lebofsky L.A., Greenberg R., Tedesco E.F., Veeder G., 1988, Icarus 75,518

Lumme K., Bowell E., 1981a, AJ 86, 1694

Lumme K., Bowell E., 1981b, AJ 86, 1705

Lumme K., Karttunen H., Bowell E., 1990, A\&A 229, 228

Magnusson P., 1986, Icarus 68, 1

Magnusson P., 1990, Icarus 85, 229 
Magnusson P., Barucci M.A., Drummond J.D., et al., 1989, in Tancredi G., Gallardo T., 1991, A\&A 242, 279

Asteroids II, Binzel R.P., Gehrels T. and Matthews M.S. Taylor R.C., 1979, in Asteroids I, Gehrels T. (ed.). Univ. of (eds.), p. 66

McCheyne R.S., Eaton N., Meadows A.J., 1985, Icarus 61, 443

Michalowski T., 1993, Icarus 106, 563

Michalowski T., Velichko F.P., 1990, Acta Astron. 40, 321

Michalowski T., Velichko F.P., Di Martino M., et al., 1995, Icarus 118, 292 Arizona press, Tucson, p. 480

Taylor R.C., Birch P.V., Pospieszalska-Surdej A., Surdej J., 1988, Icarus 73, 314

Tedesco E.F., 1989, in Asteroids II, Binzel R.P., Gehrels T. and Matthews M.S. (eds.), p. 1090

Tedesco E.F., Zappala V., 1980, Icarus 43, 33

Nielsen R.F., 1983, Inst. Theor. Astrophys. Oslo Report 59, Tholen D.J., 1989, in Asteroids II, Binzel R.P., Gehrels T. and Hauge O. (ed.), p. 141 Matthews M.S. (eds.), p. 1139

Rodríguez E., González-Bedolla S.F., Rolland A., Costa V., López de Coca P., 1997, A\&A 324, 959

Rodríguez E., Rolland A., López-González M.J., Costa V., 1998, A\&A 338, 905

Scaltriti F., Zappala V., 1977, A\&AS 30, 169

Warren W.H., Hesser J.E., 1977, ApJS 34, 207

Weidenschilling S.J., Chapman C.R., Davis D.R., et al., 1987, Icarus 70, 191

Zappala V., Di Martino M., Cacciatori C., 1983, Icarus 56, 319 